\title{
HPeak: an HMM-based algorithm for defining read-enriched regions in ChIP-Seq data
}

\author{
Zhaohui S Qin*1,2,3, Jianjun Yu3,4, Jincheng Shen'1, Christopher A Maher2,3,4, Ming Hu1', Shanker Kalyana-Sundaram³, \\ Jindan Yu ${ }^{5}$ and Arul M Chinnaiyan2,3,4,6,7,8
}

\begin{abstract}
Background: Protein-DNA interaction constitutes a basic mechanism for the genetic regulation of target gene expression. Deciphering this mechanism has been a daunting task due to the difficulty in characterizing protein-bound DNA on a large scale. A powerful technique has recently emerged that couples chromatin immunoprecipitation (ChIP) with next-generation sequencing, (ChIP-Seq). This technique provides a direct survey of the cistrom of transcription factors and other chromatin-associated proteins. In order to realize the full potential of this technique, increasingly sophisticated statistical algorithms have been developed to analyze the massive amount of data generated by this method.

Results: Here we introduce HPeak, a Hidden Markov model (HMM)-based Peak-finding algorithm for analyzing ChIPSeq data to identify protein-interacting genomic regions. In contrast to the majority of available ChIP-Seq analysis software packages, HPeak is a model-based approach allowing for rigorous statistical inference. This approach enables HPeak to accurately infer genomic regions enriched with sequence reads by assuming realistic probability distributions, in conjunction with a novel weighting scheme on the sequencing read coverage.

Conclusions: Using biologically relevant data collections, we found that HPeak showed a higher prevalence of the expected transcription factor binding motifs in ChIP-enriched sequences relative to the control sequences when compared to other currently available ChIP-Seq analysis approaches. Additionally, in comparison to the ChIP-chip assay, ChIP-Seq provides higher resolution along with improved sensitivity and specificity of binding site detection. Additional file and the HPeak program are freely available at http://www.sph.umich.edu/csg/qin/HPeak.
\end{abstract}

\section{Background}

Understanding transcriptional regulation is essential to deciphering the genetic pathways involved in various cellular processes and represents one of the major challenges in molecular biology. One critical step during this process is to determine how proteins interact with target DNA to regulate gene expression. Chromatin immunoprecipitation (ChIP) followed by PCR amplification of specific target DNA has been the primary approach to detect in vivo protein-DNA interaction [1,2]. However, the ChIP-PCR assay has been limiting in characterizing ChIP-enriched genomic DNA on a genome scale. To address this, various techniques have been developed to

\footnotetext{
* Correspondence: qin@umich.edu

${ }^{1}$ Center for Statistical Genetics, Department of Biostatistics, School of Public Health, University of Michigan, 1415 Washington Heights, Ann Arbor, MI 481092029, USA

Full list of author information is available at the end of the article
}

identify the binding sites of specific DNA-associated proteins [3]. One frequently used technique is ChIP-chip [46], in which the protein-bound DNA is detected through hybridization to DNA microarrays containing a fixed set of probes. However, this approach is heavily biased towards the predetermined probes selected on the DNA microarray, limiting the scale and resolution of this method.

More recently, ChIP-Seq, leveraging massively parallel next-generation sequencing technology, has emerged as a powerful method for genome-wide mapping of proteinDNA interactions and histone modifications [7-9]. Using this technology, numerous studies have been conducted to characterize the genomic landscape of various transcription factors (TFs), histone marks and methylation patterns [10-19]. In ChIP-Seq experiments, the ChIP process isolates DNA fragments bound by a protein using a corresponding antibody. Oligonucleotide adapters are 
then linked to the DNA to allow ultra-high-throughput sequencing. Through direct sequencing of all of the ChIP-enriched DNA fragments, ChIP-Seq is capable of revealing protein-DNA interaction sites across the entire genome, making it a valuable tool for researchers.

An array of computer algorithms has been developed to analyze ChIP-Seq data aiming to identify ChIP-enriched regions [10,11,20-31]. Excellent reviews of these methods can be found in Spyrou et al. 2009 [28] and Laajala et al. 2009 [32]. A brief description of the seven methods chosen for comparison in this study can be found in the Method section. Although performed well in ChIP-Seq studies, the majority of these methods are rule-based therefore lack the ability to determine the significance of each region. To address this, we have adopted a probability model-based approach to explicitly model noise within sequencing data, thereby enabling rigorous statistical inference. For example, the probability of enrichment can be derived and used to compare across samples and experiments. Our approach, referred to as HPeak, utilizes a hidden Markov model (HMM). HMMs have been successfully applied for the analysis of ChIP-chip data [33-37], which motivated us to adopt HMM in our present algorithm. Recently, Mikkelsen et al. (2007) [12] and $\mathrm{Xu}$ et al. (2008) [24] have utilized HMMs in their ChIP-Seq studies. However, very little detail of their HMM is provided in Mikkelsen et al. and the ChIPDiff method presented in $\mathrm{Xu}$ et al. is restricted to analyzing comparative histone modification data. By using a novel unbalanced weighting scheme, HPeak will account for the uncertainties in the actual lengths of DNA fragments. Therefore, it is capable of accurately reconstructing the genome-wide coverage profiles of DNA fragments. Such information can be used to define the boundaries of ChIP-enriched regions, which is indicated by the significantly elevated DNA fragment coverage relative to the neighboring genomic regions. Overall, we demonstrated that HPeak produces higher motif enrichment in the peaks identified without sacrificing sensitivity when compared with other existing peak-calling algorithms.

\section{Results}

\section{Datasets}

To demonstrate the performance of the HPeak algorithm, in this study we used four previously published ChIP-Seq data sets including the NRSF (neuronrestrictive silencer factor) dataset [10], the STAT1 (signal transducer and activator of transcription protein 1) dataset [11] and datasets from two histone marks H3K4me3 and H3K27me3 [8]. We selected these two histone mark datasets because both H3K4me3 and H3K27me3 are important histone modifier and they show opposite modification patterns [13].
The NRSF ChIP-Seq data [10] was downloaded from a website of Illumina (Illumina, Inc. San Diego, CA), now accessible from GEO with accession number GSE13047. It consists of two files of $25 \mathrm{bp}$ sequencing reads in ELAND output file format. Only reads that uniquely mapped to the human reference genome, with up to two mismatches, were included in these two files. One file has about 1.7 million reads that were obtained from the sample treated by ChIP. The other file contains about 2.3 million reads obtained from the mock control sample.

The STAT1 dataset [11] was downloaded from http:// www.bcgsc.ca/data/chipseq. It consists of two files in ELAND output file format, each of which contains reads combined from six lanes of a flowcell. The lengths of reads contained in these two files range from 21 to $27 \mathrm{bp}$. Only reads that uniquely mapped to the human reference genome, with up to two mismatches, were included in these two files. The numbers of uniquely-mapped reads contained in these two files are 15.3 and 13.0 million for the stimulated and the unstimulated samples respectively.

The BED format of aligned reads obtained from H3K4me3 and H3K27me3 ChIP-Seq experiments [8] were downloaded from http://dir.nhlbi.nih.gov/papers/

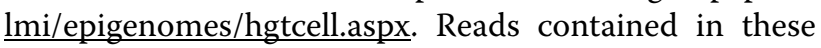
two files are $24 \mathrm{bp}$ in length. The numbers of available reads contained in these two files are 16.8 and 9.0 million respectively.

\section{ChIP-Seq reproducibility}

It is of critical importance to examine the reproducibility of an experimental assay to confirm that it returns consistent results on biological and technical replicates. In this study, we evaluated the reproducibility of ChIP-Seq by examining the similarity of genome-wide distributions of sequencing reads obtained from duplicated or distinct samples. A common strategy is to apply a Chi-square test of homogeneity to compare the two distributions; however, it faces the challenge of selecting the quantity and size of intervals to cover the genome. In this study, we took an alternative approach using the two-sample Kolmogorov-Smirnov (K-S) test to evaluate the distribution of sequencing reads across chromosomes. To compare under the same setting, we first separated reads into individual chromosomes and forward/reverse strands. Under the null hypothesis that the two samples are reproducible, the genome-wide distributions of sequencing reads are assumed to be identical. Thus we expect non-significant outcome from the K-S test conducted in each chromosome/strand combination. Bonferroni correction was used to correct for multiple testing.

For our reproducibility analysis we used the STAT1 ChIP-Seq data [11], as this dataset is comprised of reads from multiple lanes. In total, there are six lanes each for the stimulated and unstimulated samples. The variance 
between the numbers of usable reads differed substantially between lanes, ranging from 0.7 million to 4.3 million reads per lane of stimulated sample, and 0.6 million to 3.4 million per lane of unstimulated sample. Since differences in sequencing depth affect the reproducibility assessment, we only considered pairs of lanes with similar numbers of uniquely mapped reads (within $10 \%$ ). Table 1 lists the numbers of chromosome/strand combinations in which the p-value from the K-S test is lower than the Bonferroni corrected significance threshold of 0.001 . As expected, we found that no more than one out of 48 chromosome/strand combinations showed significant discrepancy for a pair of lanes within either the stimulated or the unstimulated samples. When comparing lanes from the stimulated group to the unstimulated group, we observed a much higher frequency of chromosome/ strand combinations displaying significant discrepancies (36 to 42 out of 48). Figure S1 in additional file 1 shows plots of empirical cumulative distribution functions (ECDFs) under various scenarios. These observations led us to conclude that the K-S test, along with the ECDF plots, provide a rigorous quantitative means for assessing reproducibility in the ChIP-Seq assay.

\section{Comparison of ChIP-Seq with ChIP-chip data}

One question that arose from the analysis of the ChIPSeq experiment is how well it performs relative to the ChIP-chip experiment. We selected STAT1 for a comparison study, since it has been recently evaluated using the ChIP-chip technique [38]. Using a threshold of false discovery rate (FDR) [39] 0.05, Euskirchen et al. identified

Table 1: Summary of reproducibility from two-sample Kolmogorov-Smirnov tests performed on the STAT1 ChIP-Seq data*.

\begin{tabular}{llll}
\hline & \multicolumn{3}{c}{ Stimulated } \\
\hline Lanes & $1: 2$ & $5: 6$ & $7: 8$ \\
Significant & 0 & 0 & 0
\end{tabular}

\begin{tabular}{llll}
\hline & \multicolumn{3}{c}{ Unstimulated } \\
\hline Lanes & $2: 5$ & $3: 4$ & $7: 8$ \\
Significant & 1 & 1 & 0 \\
& & & \\
\hline
\end{tabular}

Stimulated vs unstimulated

\begin{tabular}{llllll}
\hline Lanes & $1: 3$ & $1: 4$ & $2: 3$ & $2: 4$ & $5: 7$ \\
Significant & 38 & 40 & 42 & 40 & 36 \\
\hline
\end{tabular}

*numbers displayed in the table are quantities of chromosome/ strand combinations that show significant discrepancy when conducting the two-sample Kolmogorov-Smirnov test.
3,701 ChIP-enriched regions in about one-tenth of the genome, mostly on chromosomes 20, 21, 22, X and Y. For ease of comparison, we focused only on ChIP-Seq peaks located within these five chromosomes, which corresponds to 2,023 out of 24,394 regions. A motif scan demonstrated that the peaks defined by ChIP-Seq showed greater enrichment of the STAT1 motif than peaks defined by ChIP-chip. Comparison of Chi-square statistics using the ChIP-Seq data showed that motifs in the STAT family are much more enriched relative to motifs from other families (the negative log transformed p-value for STAT family is about twice as large as the second highest) whereas the STAT motif family is not the most enriched in the ChIP-chip data (Figure 1A, B). Our results supported earlier reports of the superior resolution of the ChIP-Seq technique [10].

It has been reported that regions with higher ChIP-chip scores are more likely to contain the motif(s) of interest [40]. A natural question is whether the ChIP-Seq data shows a similar property. To investigate this, we ranked all peaks identified by HPeak from the STAT1 ChIP-Seq data according to the average posterior probabilities of all bins within the peak. We then divided these peaks into five groups of equal sizes. We next calculated the Chisquare test statistics of motif enrichment within each of these five groups of sequences relative to the lengthmatched control sequences. For comparison, we performed the same analysis on regions identified by the ChIP-chip study on STAT1 [38]. We found that the change in motif enrichment is more dramatic among regions identified by ChIP-Seq than ChIP-chip (Figure $1 C)$.

\section{Performance comparison with other ChIP-Seq peak-calling algorithms}

To demonstrate the performance of HPeak, we tested it on the four publicly available ChIP-Seq datasets described above. Seven ChIP-Seq analysis packages were used in this comparison, PeakFinder [10], FindPeak $[11,30]$ MACS [20], SISSRs [23], CisGenome [22], ChIPseeqer http://physiology.med.cornell.edu/faculty/elemento/lab/chipseq.shtml and ChIPDiff [24] (only used for analyzing histone mark data). We applied these software programs to the datasets using either the default or the recommended parameters according to the program manuals.

Since FindPeaks analyzes treated and control samples separately. To provide a fair comparison, we ran HPeak twice, first using both treated and control samples and second with the treated sample only (comparable to FindPeaks).

A brief summary of the peak-calling results of the NRSF ChIP-Seq data is presented in Table 2. The number of identified peaks ranges from 1,935 (Peak Finder) to 
A.

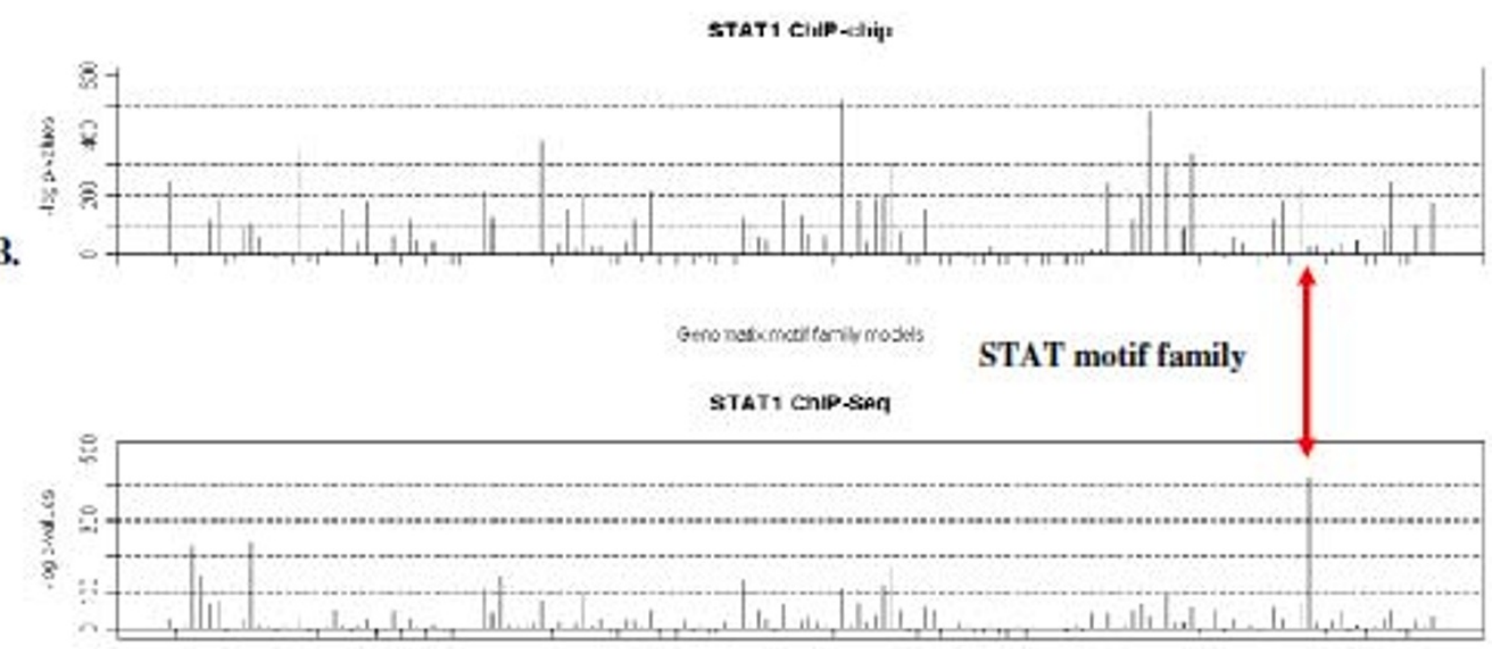

C.

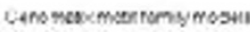

STAT1 ChIP-Seq and ChIP-chip

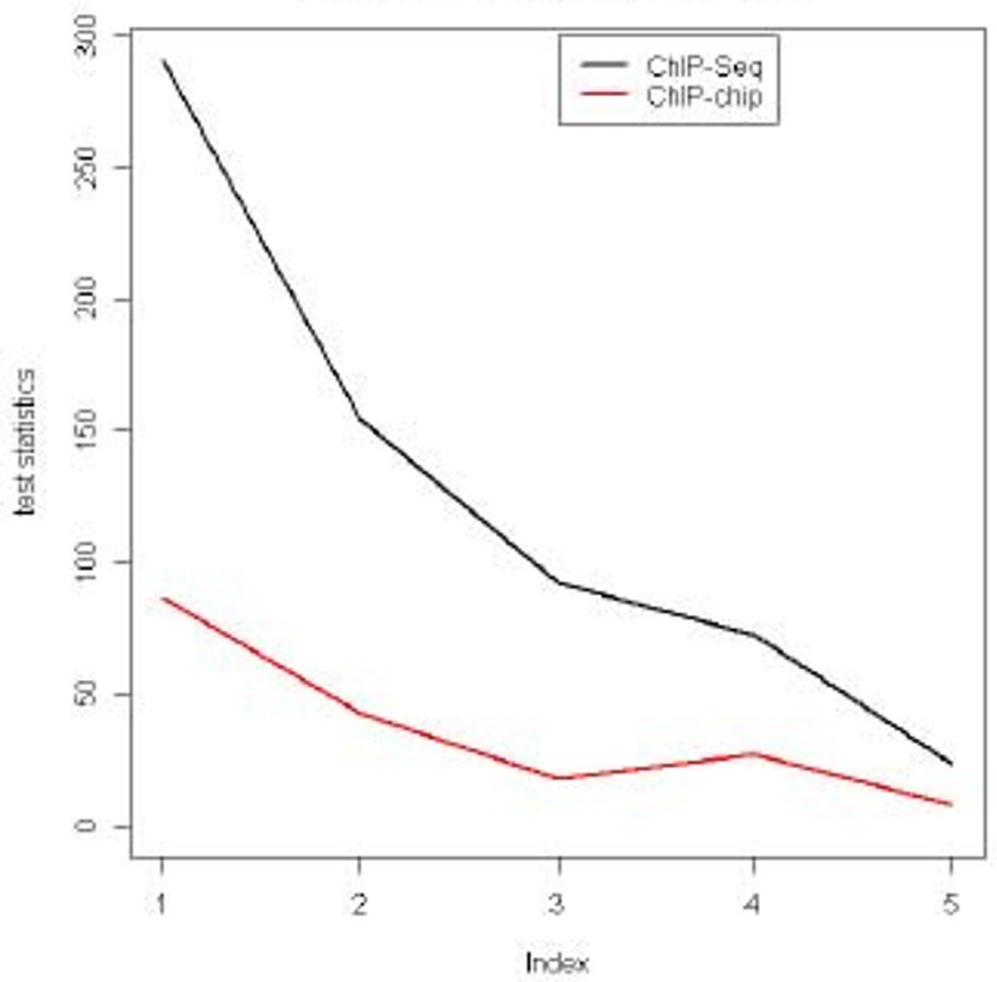

Figure 1 Comparison of motif enrichment in peaks identified by ChIP-chip and Chip-Seq. Chi-square test statistics from $2 \times 2$ contingency table is shown for all 153 families of vertebrate TF binding motif patterns found in the MatBase library 7.0 database of Genomatix (Genomatix, GmBH, Munich, Germany). Motif scan was performed using MatInspector in Genomatix using the default setting. A. STAT1 ChIP-chip result (on about $10 \%$ of the entire genome, the majority of them (88\%) on chromosomes 20,21,22, X and Y). B. STAT1 ChIP-Seq result (subset of 2,023 peaks out of 24,394 located on Chromsome 20, 21, 22, X and Y). C. Correlation between motif enrichment and rank of significance in peaks indentified from STAT1 ChIP-Seq and ChIP-chip experiments. All peaks were ordered according to their significance and then divided into five segments of equal sizes. Their motif enrichment is measured by Chi-square test statistics in these five segments are shown from left to right. 
5,243 (SISSRs). As explained in the Method section, we used motif enrichment as the measure of performance to evaluate the results obtained from different ChIP-Seq analysis programs. The motif enrichment in the ChIPenriched and random control sequences was measured by Chi-square test statistics derived from $2 \times 2$ contingency tables (see the Methods section). Since the numbers of peaks identified differed substantially across these methods, for a fair comparison, we ranked the peaks by each method s specific significance criterion (score or number of reads in peaks), and subsequently selected the top $m$ peaks nominated from each approach to compare their motif enrichment (Figure 2A). Overall, for the NRSF dataset, we found HPeak performs the best in most cases when both the stimulated and unstimulated samples were used. When only the stimulated sample was used, HPeak outperformed FindPeaks in terms of enrichment of the expected motif.

Next, we compared motif enrichment in peaks identified by one method, but not the other, in order to determine the distinguishing features of each program. We found that, among the 2,323 peaks that were identified as significant by HPeak but not PeakFinder, the NRSF motif is the most enriched relative to length-matched random control sequences (Chi-square test $\mathrm{p}$-value $<10-16$ ) among all 508 motifs in the MatBase matrix library 7.0. Analogously, among the 495 peaks that were identified by HPeak, but not FindPeaks, the NRSF motif is again the most enriched (Chi-square test p-value $5.8 \times 10-14)$. In contrast, among the 285 peaks that were identified by FindPeaks but not HPeak, the NRSF motif ranked 68th among the 508 motifs tested with p-value 0.012, which was no longer significant after correction for multiple testing. When tested using the two half sites of NRSF motif, which have been shown to present in NRSF ChIPSeq binding domains in statistical significant manner relative to random sequences [10], we found that the right half-site ranked 32nd with p-value of 0.0015 while the left half site ranked 213th with p-value 0.868 . Neither was significant after correction for multiple testing. Overall, this suggests that peaks identified by other programs but not by HPeak are likely to be false positives.

We next assessed the performance of HPeak using the STAT1 ChIP-Seq dataset [11]. We did not compare it to PeakFinder, since it only identified approximately onetenth of the peaks found by most of the other algorithms under its default parameter settings (modifications of the significance threshold failed to result in much increase in the number of peaks). Compared to the NRSF dataset, the STAT1 data represents a deeper coverage scenario, with an increase in usable reads obtained from multiple lanes.

For this dataset, when both stimulated and unstimulated samples were considered under the default setting,
HPeak identified 24,394 peaks. The number of peaks identified by competing algorithm ranges from 9,561 (ChIPseeqer) to 41,127 (FindPeaks). Using both the stimulated and unstimulated samples, HPeak showed the highest motif enrichment. When only the stimulated sample was used, HPeak identified 43,440 peaks. Using the stimulated sample alone, HPeak showed significantly higher motif enrichment than FindPeaks. However, adding unstimulated control samples improved HPeak's performance in terms of motif enrichment (Figure 2B). This suggests that the use of control samples may improve the accuracy of identified peak regions as substantial regionspecific biases are often observed in genome-wide sequencing due to the different accessibility of genomic regions.

In the above comparison, the lengths of the peaks differed across tested algorithms. Therefore, if we scan these peaks only, those with wider peaks will contain more motif of interest. We have corrected such bias by introducing size-matched control sequences. An alternative solution to remove the size bias is to search a fixed window around the peak summit. This method was adopted in the MACS study [20]. We have examined the performance using this method as well. The results of these scans can be found in Figure S2 in additional file 1. From there, we found that nearly all methods reported similar number of motif occurrences in the $200 \mathrm{bp}$ regions around the peak summits especially for the NRSF data. MACS showed slight edge perhaps owing to its accurate positioning of the peak summits.

For the two histone mark datasets, since there is no list of gold standard binding loci, and there are no known motifs associated with either mark, we tabulated the sets of peaks identified from all peak-calling algorithms and studied the overlap among these sets of peaks. The results are summarized in Tables 2 and 3. We found that results from most of the peak-calling algorithms we tested are very similar especially for the open chromatin histone mark H3K4me3 (most overlap percentages are greater than 90\%). For H3K4me3, the overlap pattern is similar to what we observed for TFs NRSF and STAT1 (Table S1 in additional file 1). For H3K27me3, the percentages of overlap vary greatly, most likely due to the fact that the numbers of peaks generated by these peakcalling algorithms are quite different. We did not included results from ChIPDiff in this evaluation since this program produces many more peaks than others and ChIPDiff was originally designed for the comparison between two cell types.

In addition, we observed that the patterns of the peaks identified from ChIP-Seq experiments on TFs like NRSF and STAT1 are different from those obtained from ChIPSeq experiments on histone marks like H3K4me3 and H3K27me3. The average peak length is $253 \mathrm{bp}$ for NRSF 
Table 2: Summary of peaks identified by various peaking calling algorithms.

\begin{tabular}{|c|c|c|c|c|c|c|c|c|}
\hline & Peak Findera & MACS & HPeak $^{b}$ & FindPeaks & $\begin{array}{c}\text { HPeak } \\
\text { (chip only) }\end{array}$ & $\begin{array}{c}\text { ChIPseeqer } \\
\text { (control) }\end{array}$ & SISSRs & CisGenome \\
\hline \multicolumn{9}{|l|}{ NRSF } \\
\hline \multicolumn{9}{|l|}{ chip: $1.7 \mathrm{M}$} \\
\hline \multicolumn{9}{|l|}{ mock: $2.3 \mathrm{M}$} \\
\hline Number of peaks & 1,935 & 4,679 & 4,404 & 3,445 & 4,085 & 2,361 & 5,243 & 2,545 \\
\hline Covered space (kb) & 908 & 1,902 & 1,112 & 4,936 & 1,512 & 682 & 276 & 775 \\
\hline Avg peak width (bp) & 469 & 406 & 253 & 1,433 & 370 & 289 & 53 & 304 \\
\hline \multicolumn{9}{|l|}{ STAT1 } \\
\hline \multicolumn{9}{|l|}{ stimulated: $15.3 \mathrm{M}$} \\
\hline \multicolumn{9}{|l|}{ unstimulated: $13.0 \mathrm{M}$} \\
\hline Number of peaks & - & 22,402 & 24,490 & 41,127 & 43,443 & 11,662 & 9,561 & 38,878 \\
\hline Covered space (kb) & - & 16,940 & 6,562 & 46,781 & 15,354 & 3,025 & 455 & 10,012 \\
\hline Avg peak width (bp) & - & 756 & 269 & 1,137 & 353 & 259 & 48 & 258 \\
\hline \multicolumn{9}{|l|}{ H3K4me3 } \\
\hline \multicolumn{9}{|l|}{$16.8 \mathrm{M}$} \\
\hline Number of peaks & 28,960 & 27,568 & - & 33,890 & 41,217 & 31,773 & 137,286 & 46,261 \\
\hline Covered space (kb) & 30,610 & 36,675 & - & 83,348 & 30,435 & 18,789 & 6,464 & 26,500 \\
\hline Avg peak width (bp) & 1,057 & 1,330 & - & 2,459 & 738 & 591 & 47 & 573 \\
\hline \multicolumn{9}{|l|}{ H3K27me3 } \\
\hline \multicolumn{9}{|l|}{$9.0 \mathrm{M}$} \\
\hline Number of peaks & 335 & 1,342 & - & 8,348 & 4,858 & 417 & 2,458 & 437 \\
\hline Covered space (kb) & 83 & 607 & - & 19,234 & 894 & 115 & 138 & 191 \\
\hline Avg peak width (bp) & 248 & 452 & - & 2304 & 184 & 276 & 56 & 436 \\
\hline
\end{tabular}

and 269 bp for STAT1 respectively. In contrast, peaks of H3K4me3 are much broader (738 bp) with high variance $\left((731 \mathrm{bp})^{2}\right)$ while peaks of H3K27me3 are shorter (183 bp) with lower variance $\left((145 \mathrm{bp})^{2}\right)$. Histograms of peak lengths from the four ChIP-Seq datasets can be found in Figure S3 in additional file 1.

\section{Discussion}

In this study we have described HPeak, an HMM-based algorithm for defining ChIP-enriched peaks from short sequencing read data generated from ChIP-Seq experiments. Distinct from various algorithms currently available [10,11,20-31], HPeak explicitly assumes probability distributions to model coverage profiles of hypothetical DNA fragment (HDFs, see the Methods section) along the genome. After dividing each chromosome into bins, HPeak employs an HMM to distinguish ChIP-enriched regions from the background. Generalized Poisson (GP) [41] or zero inflated Poisson (ZIP) [42] distributions were used to model observed HDF counts in each bin, allowing for a more optimal fit to the data than a standard Poisson distribution. The end of each HDF was down-weighted when evaluating coverage to account for the uncertainties in ChIP DNA fragment length. These features facilitate the recognition of the core regions that show significant ChIP-enrichment. As a result, HPeak produces more calibrated peaks with higher motif concentration when compared to other peak-finding algorithms. Evaluation of experimental data showed favourable performance in terms of motif enrichment. Because the underlying HMM is quite general, HPeak may be applied to a wide spectrum of ChIP-Seq data with different experimental design and different sequencing depth, achieving balanced sensitivity and specificity with little or no fine-tuning by the users. In a recent study, Laajala et al. conducted a comprehensive performance comparison of existing peak-calling software [32]. HPeak was included in that study along with eight other published peak-calling algo- 


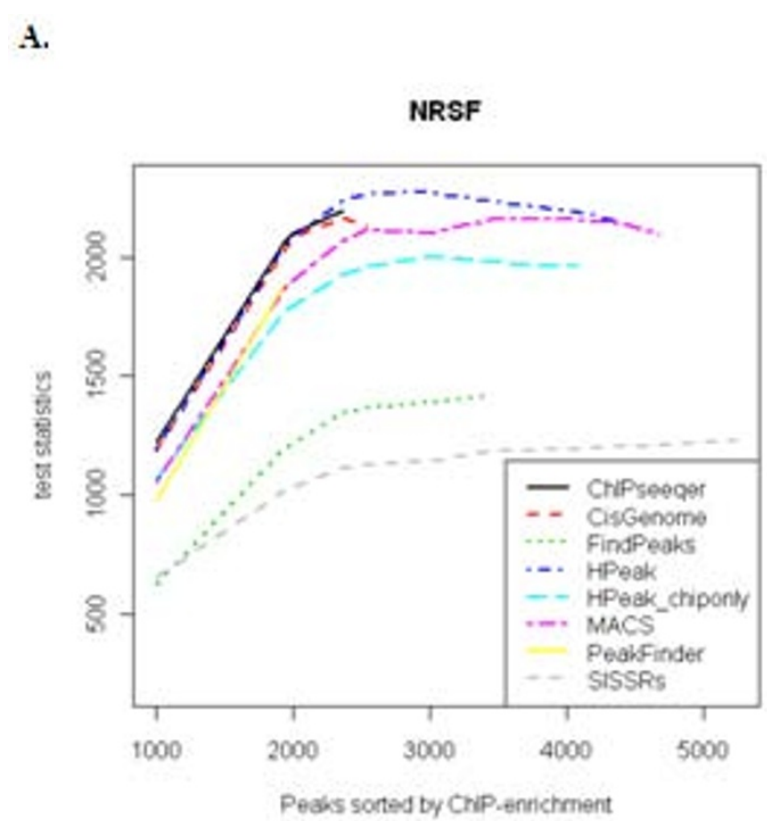

B.

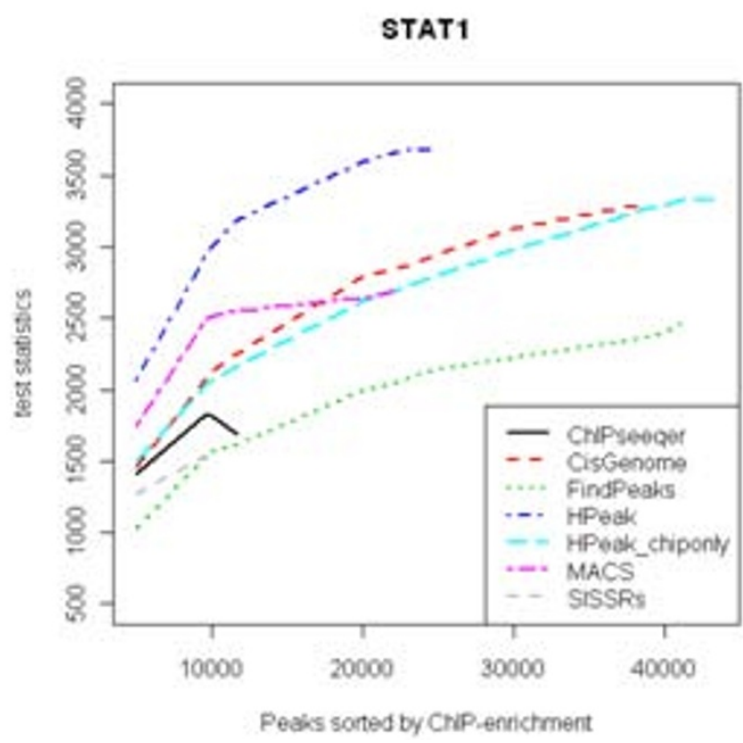

Figure 2 Performance comparison between HPeak (using data from both treated and untreated samples or using data from treated sample only) and other ChIP-Seq analysis algorithms. A NRSF ChIP-Seq data: Chi-square test statistics of motif enrichment comparing original sequences under peaks and a set of random control sequences. B. STAT1 ChIP-Seq data: Chi-square test statistics of motif enrichment comparing original sequences under peaks and a set of random control sequences.

rithms. We noticed that overall HPeak performance is quite encouraging. For example, HPeak showed the best true positive rate and is closest to the optimum when testing on the NRSF ChIP-Seq data. This is consistent with what we have found.
A key advantage of model-based methods is that they are compatible with rigorous statistical inference. For example, under our model assumption, we can directly calculate the probability of observing the actual number of HDFs in a bin. Such probabilities can then be used to rank all peaks identified. This is important, as we have shown that higher-ranking peaks are more likely to harbour canonical binding motifs (Figure 1C). Furthermore, these probabilities can facilitate comparison of peaks across samples and studies. Another advantage of modelbased method is that additional information such as GC content and mappability scores can be easily incorporated by extending the model.

In addition to its ability to identify the ChIP-enriched portion of the genome, HPeak provides more extensive information than other available programs. For example, as an option to users, HPeak provides more comprehensive annotation corresponding to each peak such as GC content, phylogenetic conservation (phastCons scores [43]), genomic features of the region (exon, intron, 5' UTR, 3' UTR, intergenic), and distance to the TSS of nearby genes. HPeak also provides an optional WIG file containing the genomic locations of all identified peaks, easily enabling the visualization of all of the peaks within the UCSC genome browser. Further, HPeak provides an optional FASTA format sequence file containing nucleotide sequences of all peaks to facilitate subsequent motif analysis.

When comparing publicly available STAT1 ChIP-Seq and ChIP-chip data, we found that the ChIP-Seq technique has a clear advantage over the ChIP-chip technique in terms of enriching for an expected motif under the predicted peaks. The improvement can be largely attributed to the increased resolution offered by the new sequencing technology. By enabling the detection of narrower peaks flanking the true binding sites, ChIP-Seq reveals a higher concentration of the predicted binding motif within its peaks. It is known that the significance measure derived from the ChIP-chip data is correlated with the probability that a region contains the canonical binding motif [40]. We found that such correlation is much stronger in ChIP-Seq data (Figure 1C). This implies that the read coverage profile is very informative on the presence and location of actual functional binding sites.

In this study, rather than the commonly used Chisquare goodness-of-fit test, we proposed to use the K-S test as an alternative to evaluate the reproducibility of datasets obtained under different conditions. We found that the K-S test is better suited for sequencing data than the Chi-square goodness-of-fit test, since there is no need to divide chromosomes into windows and correlation/ reproducibility can be conveniently visualized by plotting ECDFs (Figure $\mathrm{S} 1$ in additional file 1). 
Table 3: Summary of overlaps among peaks identified by different peaking calling algorithms in H3K4me3 and H3K27me3 ChIP-Seq datasets*.

\begin{tabular}{|c|c|c|c|c|c|c|c|}
\hline H3K4me3 & & & & & & & \\
\hline Method (\# of peaks) & $\begin{array}{l}\text { Peak } \\
\text { Finder }\end{array}$ & MACS & HPeak & Find Peaks & $\begin{array}{l}\text { ChIP } \\
\text { seeqer }\end{array}$ & SISSRs & CisGenome \\
\hline Peak Finder $(28,960)$ & & 84.3 & 99.8 & 100.0 & 98.2 & 98.0 & 99.6 \\
\hline MACS $(27,568)$ & & & 90.6 & 95.4 & 84.8 & 87.7 & 84.8 \\
\hline HPeak $(41,217)$ & & & & 99.4 & 100 & 98.9 & 97.1 \\
\hline FindPeaks $(33,886)$ & & & & & 100 & 99.8 & 100 \\
\hline ChIPseeqer $(31,773)$ & & & & & & 99.0 & 100 \\
\hline SISSRs $(137,286)$ & & & & & & & 94.6 \\
\hline \multicolumn{8}{|l|}{ CisGenome $(46,261)$} \\
\hline \multicolumn{8}{|l|}{ H3K27me3 } \\
\hline Method (\# of peaks) & $\begin{array}{l}\text { Peak } \\
\text { Finder }\end{array}$ & MACS & HPeak & Find Peaks & $\begin{array}{l}\text { ChIP } \\
\text { seeqer }\end{array}$ & SISSRs & CisGenome \\
\hline Peak Finder (335) & & 44.8 & 99.7 & 100 & 77.3 & 90.4 & 70.9 \\
\hline MACS $(1,341)$ & & & 42.5 & 42.4 & 23.5 & 24.4 & 32.9 \\
\hline HPeak $(4,858)$ & & & & 72.1 & 77.7 & 98.2 & 82.0 \\
\hline FindPeaks $(8,346)$ & & & & & 77.7 & 91.6 & 87.1 \\
\hline ChIPseeqer (417) & & & & & & 58.5 & 75.8 \\
\hline SISSRs $(2,455)$ & & & & & & & 25.4 \\
\hline CisGenome (437) & & & & & & & \\
\hline
\end{tabular}

It is worth pointing out that it is challenging to determine criteria to evaluate the performance of various peak-calling algorithms on experimental ChIP-Seq data. This is because in general very little information is available on true positive and true negative loci. We choose to use the prevalence of known motifs as a metric for performance. One caveat of this approach is that, as pointed out in $\mathrm{Hu}$ et al. 2010 [44], many of the motif patterns stored in the database may not be accurate and there maybe novel motifs that do not exist in motif databases. We speculate that the inaccurate motif patterns will affect the results of all peak-calling algorithms equally, but the actual effect remains to be seen and further studies seem warranted. Additionally, our method is not well-suited for quantifying false detection rate therefore some methods maybe put in a disadvantaged position in our comparison. Because of this, our performance evaluation results should be interpreted with caution.

The ChIP-Seq technology can be applied to other types of proteins in addition to TFs. For example, multiple studies have utilized ChIP-Seq to identify histone modification sites in the genome [12-14], which is reviewed in Park [45]. Since the underlying two-component HMM is quite general, HPeak can also be applied to data collected from other types of sequencing-based experiments such as MeDIP-seq [46], RNA-seq [47] and methylation pattern discovery [19]. In these experiments, HPeak can be used to identify regions in the genome that is significantly enriched for sequencing reads. Some adjustment of HPeak parameters such as bin size may be needed when analyzing non-ChIP-Seq type of data.

The HMM used in HPeak assumes two different states: enriched and non-enriched. Although such a scheme is well-accepted in ChIP-chip analyses [33], it is possible and of interest to consider more sophisticated HMM schemes where more than two states are allowed. As an example, we may consider a four-state HMM: enrichment of reads on the positive strand, enrichment of reads on the negative strand, enrichment of reads on both strands and no enrichment. By utilizing strand information, we 
will be able to better identify true binding events since a symmetric pattern among reads with different strands is expected around the binding sites. Another possibility is to distinguish shapes of peaks, such as sharp peaks, broad and low plateaus. These may help us to distinguish different types of binding events.

We assume HDF counts follow ZIP and GP distributions in background and ChIP-enriched regions respectively. Other probability distributions such as negative binomial (NB) has also been used to model ChIP-Seq data $[22,28]$. It is of interest to understand whether these distributions fit observed ChIP-Seq data better than the standard Poisson distribution. To investigate, we fit GP, Poisson and NB distributions to the number of HDFs found in NRSF and STAT1 ChIP-Seq data. For the number of HDFs found in the ChIP-enriched regions, we found that the GP distribution shows a much better fit than the Poisson distribution and a slightly better fit than the NB distributions. An example of the model fit can be found in Figure S4 in additional file 1. For the number of HDFs found in background regions, we found that the ZIP distribution produces a slightly better fit than both Poisson and NB distributions (data not shown).

The current HPeak algorithm does not distinguish reads of different orientation within a peak. Such information has been shown to be informative in pinpointing the summit of the peak and to estimate the DNA fragment length $[20,21,25,48]$. We plan to incorporate such information in the future release of HPeak and we believe it will further enhance the performance of the HPeak program.

\section{Conclusions}

Based on our study, we believe that HPeak will be highly useful to researchers conducting ChIP-Seq experiments. For instance, HPeak has already been utilized in a recent study to map the genomic landscape of master transcriptional regulators of prostate cancer [49]. We envision that this tool will greatly facilitate the rapid and accurate analysis of the emerging ChIP-Seq data generated by the research community.

\section{Methods}

\section{HPeak scheme}

The goal of ChIP-Seq analysis is to partition the genome into ChIP-enriched and non-enriched segments based on the number of mapped sequencing reads, such that the enriched portion of the genome is much more likely to harbour protein-DNA interaction sites. The entire procedure of HPeak analysis can be summarized into four steps (Figure 3).

In the initial step, HPeak imports the genomic coordinates of all mapped sequencing reads. Various ELAND output format (Illumina Inc, San Diego, CA) and BED formats are allowed. In order to represent the entire DNA fragment, we followed the method used by Robertson et al. [11] to extend each short read directionally from its start position to form a hypothetical DNA fragment (HDF), mimicking the ChIP DNA fragment from which the sequencing read was generated.

In the second step, HPeak partitions the entire genome into small bins of fixed length ( $25 \mathrm{bp}$ in this study) and subsequently counts the numbers of HDFs that fall in each bin throughout the genome. By adopting a bin approach, we are able to simplify computation and facilitate straightforward comparison across samples while obtaining a high resolution genome-wide ChIP DNA coverage profile. The size of the bins can be adjusted to balance the computational run time with resolution.

In the third step, HPeak applies a two-state HMM on the HDF coverage profile to distinguish blocks of consecutive ChIP-enriched bins from the background. GP and ZIP distributions were utilized to model the numbers of HDFs found in ChIP-enriched and non-enriched bins.

In the last step, HPeak generates additional output files based on the peaks called in the previous step. These include a wiggle (WIG) format file for visualization, a sequence file for subsequent motif analysis and an annotation file that details the genomic properties of each peak identified.

\section{Down-weighting the end of HDF to account for length uncertainty}

In ChIP-Seq experiments, a size selection step is employed during sample preparation to restrict the size of DNA fragments to be sequenced to a certain range (for example, $200 \mathrm{bp} \pm 25 \mathrm{bp}$ ). Since only the beginning or end portion of each DNA fragment is sequenced, we employ a read extension step described earlier in order to quantify the actual DNA fragment coverage at any given locus. To account for the uncertainties in the lengths of the DNA fragments, we gradually down-weight the HDF coverage contribution in bins covered by the end portion of each HDF. For example, if the DNA fragments are sizeselected to be between 175 and $225 \mathrm{bp}$, we assign one unit of coverage to the genomic locations covered by the first $175 \mathrm{bp}$ of the HDF, linearly decreasing the coverage assigned to the last $50 \mathrm{bp}$ from 1 to 0 (Figure S5A in additional file 1). We also assign partial coverage to bins with partial HDF coverage.

\section{Statistical model to define ChIP-enriched regions}

We adopted a two-state HMM to model the observed ChIP DNA fragment profile and classify the bins into either ChIP-enriched (peaks) or non-enriched (background) along the entire genome (Figure S5B in additional file 1). We employ a HMM because of the strong correlation of HDF coverage in adjacent bins. To infer the 
emission probabilities in the HMM, we used GP and ZIP distributions to model the numbers of HDFs found in the two types of bins. Both distributions were modified from and are more flexible than the standard Poisson distribution. The GP distribution was adopted for the ChIPenriched group. Due to the wide dynamic range of ChIPSeq data, the number of HDFs falling into ChIP-enriched bins varied dramatically and showed significant over-dispersion (the variances of HDF counts in ChIP-enriched bins are about ten times larger than the mean HDF counts in these bins). A standard Poisson distribution requires the mean and variance to be the same which is unrealistic for this type of data. In contrast, the GP distribution is much more flexible, since it contains two parameters and allows the variance to be greater than the mean. The probability density function of the GP distribution is

$$
\begin{aligned}
& P(Y=y \mid \lambda, \phi)= \\
& \left(\frac{\lambda}{1+\phi \lambda}\right)^{y} \frac{(1+\phi y)^{y-1}}{y !} \exp \left\{\frac{-\lambda(1+\phi \gamma)}{1+\phi \lambda}\right\},
\end{aligned}
$$

here $\varphi, \lambda>0$.

Since the majority of the genome is not enriched, there are significantly more empty bins required to model the background than would be expected from the Poisson distribution. An ideal alternative is the zero-inflated Poisson (ZIP) distribution [42], which is essentially a mixture distribution of point mass at zero and a Poisson distribution. The probability density function of the ZIP is:

$$
f(x \mid \pi, \mu)= \begin{cases}(1-\pi)+\pi e^{-\mu} & \text { if } x=0 \\ \frac{\pi e^{-\mu} \mu^{x}}{x !} & \text { if } x>0\end{cases}
$$
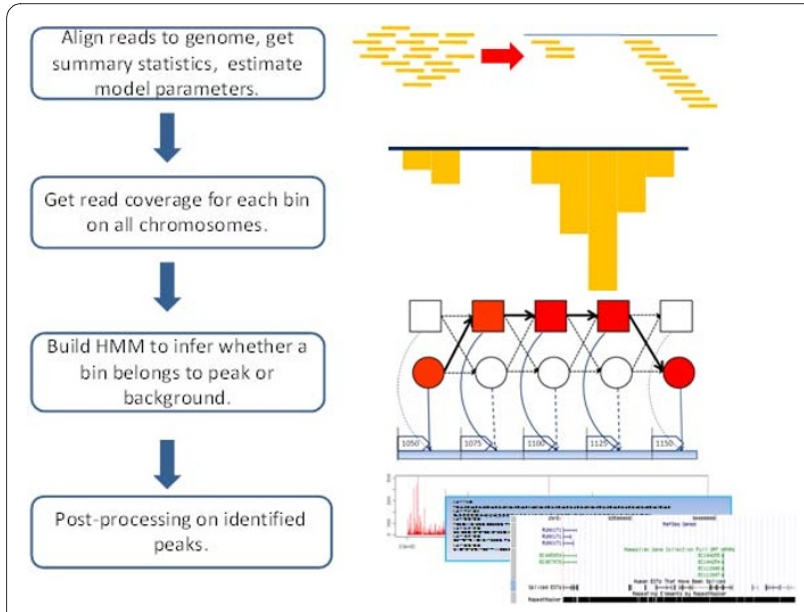

Figure 3 Workflow of HPeak analysis of ChIP-Seq data where $\pi(0 \leq \pi \leq 1)$ is the proportion of zeros in the mixture distribution. Compared to the standard Poisson distribution, the GP and ZIP distributions provide a much better fit to the observed data due to improved flexibility. This allows the assignment of accurate probabilities to each bin and defines the boundaries of the enriched regions more precisely.

Some ChIP-Seq experiments are carried out using paired samples: a treated sample (stimulated) with an untreated control (unstimulated). For the paired design, we used the bivariate GP/ZIP distributions to model the difference in HDF coverage in the two types of samples. More specifically, let $D_{i}=X_{i}-Y_{i}, i=1,2 . X_{i}, Y_{i}$ represent the HDF counts in bins residing in the treated and untreated samples respectively; $i=1$ indicates ChIPenriched; $i=2$ indicates nonenriched. Assuming that $X_{1}$ follows the GP distribution while $X_{2} Y_{1}$ and $Y_{2}$ follow ZIP distributions, we calculate the marginal probabilities of observing the HDF coverage differences between the two samples in the same bin, based on the bivariate distributions using the parameters estimated within treated or untreated samples separately. An HMM is then designed to perform inference on the enriched/non-enriched states.

\section{HMM parameter estimation}

For HMM parameter estimation, we use the well-established Viterbi algorithm [50]. In the initial step, we use summary statistics to get a quick and rough estimate of the transition and emission probabilities. Following the method described in Robertson et al. [11], all HDFs that overlap are merged into a single consecutive candidate peak. The read coverage of each candidate peak is then calculated to determine whether it surpasses a significance threshold that is required for it to be classified as a ChIP-enriched peak. The initial probability of being in a peak, and the transition probability from background to peaks, is equivalent to the proportion of the genome that is covered by the peaks, as defined above. The transition probability from a peak back to the background is defined such that the a priori length of the peaks is roughly equivalent to the median length of the peaks as defined above. For instance, supposing that $L$ represents the median length of these peaks, $p_{0}$ represents the transition probability from peak to background, and $d$ is the bin size, then $p_{0}$ can be estimated by solving $\left(1-p_{0}\right)^{L / d}=1 / 2$.

The emission probabilities in the HMM are derived from the GP and ZIP distributions that are used to model the HDF count data in ChIP-enriched and non-enriched bins. The initial parameters in these distributions were estimated as follows. For the ZIP distribution that models background HDF counts, we first selected the bins with HDF counts less than $k_{\text {low }}$, where $k_{\text {low }}=k / 2$ and $k$ is the 
minimum number of HDFs found in bins belonging to ChIP-enriched peaks. Next, we used the method of moments to estimate GP distribution parameters $\varphi$ and $\lambda$, as well as ZIP distribution parameters $\mu$ and $\pi$. Detailed formulas of these estimation can be found in additional file 1. For the two-sample case, we obtain the parameters of two sets of GP and ZIP distributions from treated and untreated samples separately.

After the initial step, we iterate the two steps of the Viterbi algorithm: conditional on the current estimate of the model parameters, we derive the hidden states for each bin across the genome by evaluating which model fits the observed HDF count data better; conditional on the currently assigned hidden states, we separate all bins into ChIP-enriched and non-enriched, and update model parameters for the two categories separately. Here we no longer merge HDFs; all hidden state inferences are conducted at the bin level.

As a final result, using a user-specified posterior probability threshold, we identify blocks of consecutive bins that show significant enrichment of HDF counts from the HMM. Each set of bins is then defined as a peak. In addition to its genomic location and the length of the peak, HPeak reports the location of the highest HDF coverage within the peak, the actual maximum HDF coverage at that location, and the log transformed posterior probability of these bins being ChIP-enriched. These probabilities reflect the significance of these peaks and therefore can be used to rank the peaks.

\section{Implementation}

We have implemented HPeak in a software package using Perl and $\mathrm{C}++$. HPeak can run on most platforms including Linux, Windows and Mac OS. Using a Dell PowerEdge computer server, we found that HPeak required reasonable amounts of memory (less than $2 \mathrm{G}$ for a dataset of 15 million reads) and time (approximately 30 and 60 minutes for treated only and paired design respectively). Currently, HPeak is capable of analyzing ChIP-Seq data collected from human and mouse and can easily be extended to other species. The HPeak program is freely available at http://www.sph.umich.edu/csg/qin/HPeak.

\section{Existing peak detection algorithm}

We provide a short summary for each of the algorithms being compared in this study. For more details, please consult the original publications or the software websites.

\section{Peakfinder (ChIPSeq-mini)}

This is perhaps the earliest software for ChIP-Seq peak calling. A candidate peak is called if it contains at least $k$ reads not separated by more than $n$ bp (75 by default), and at least five of these reads are overlapping. An additional requirement is that there is at least $m$-fold enrichment (default 5-fold) when control samples are available.

\section{FindPeaks}

Findpeaks extends each read directionally to form an HDF. The length of the extension is taken to be the average length of the size-selected ChIP-DNA fragments. Subsequently overlapping HDFs are merged to form a candidate peak. A binding site is then identified if the number of HDFs in a candidate peak exceeds the significance threshold. An FDR for each binding site is estimated based on Monte Carlo simulation, which is the number of peaks identified in the randomized data divided by the number of peaks identified in the real data under the same significance threshold.

\section{MACS}

MACS first separate reads of different strands, and then empirically models the shift between the two types of strands. MACS also implements a Poisson distributionbased model to characterize the background read distribution. They termed their model a "dynamic Poisson model" to reflect the fact that the parameter of the Poisson distribution is allowed to fluctuate along the genome in order to capture local sequencing bias. MACS works with or without negative control samples. When negative control samples are available, an FDR is estimated by dividing the number of peaks identified in the control sample by the number of peaks identified in the ChIP sample.

\section{CisGenome}

CisGenome employs a sliding window strategy to identify regions with over-abundant reads. The authors assume a NB distribution for the background read occurrence which was said to provide a better fit of the data than the Poisson distribution. When negative control data are available, the authors use a binomial model to decide whether the enrichment of reads in the ChIP channel is significantly higher than in the control.

SISSRS

SISSRs first extends each read to form an HDF, next partitions the genome into windows of equal sizes, then scans the genome to count the number of reads landing in each window. Binding sites are called when the majority of reads switch from one strand to the other. A negative control sample, or a Poisson background model is used to estimate the FDR, defined to be the ratio of the number of peaks in the control sample or background model to the number of peaks observed.

\section{ChIPseeger (revised from the description provided by Dr.}

\section{Elemento)}

In this program, a read density map is first constructed by extending the reads to the average length of the DNA fragments in the sequenced DNA library and by counting the number of overlapping reads at each nucleotide position. The Poisson distribution probability model is then used to compare the observed read count to the expected read count for both ChIP and input (control) data (if 
available) and to compute a normalized peak score for each nucleotide position (based on probabilities). A peak is called when this score is greater than a specific threshold (default 15) and the interval is at least $100 \mathrm{bp}$. It is important to note that the algorithm uses the mappability map to correct for the variation in mappablity between sequences. Moreover, if input data are provided, it is further required that there is at least an $\mathrm{m}$-fold enrichment (default 2-fold) of reads in the ChIP data over the input.

\section{ChIPDiff}

ChIPDiff was developed to identify differential histone modification sites genome-wide. This method employs an HMM to infer the states of histone modification changes at each genomic location based on the observed ChIP fragment counts. To apply ChIPDiff, it is required that ChIP-Seq data from two sources (two different cell lines, etc) are available.

\section{Performance evaluation metrics Motif enrichment analysis}

Since consensus motifs are often enriched in the binding sites of the TF, motif enrichment may serve as a measure of the performance (sensitivity and specificity) when comparing ChIP-Seq peak-calling algorithms. One strategy is to scan all sequences identified as ChIP-enriched (referred to as test sequences) and compare the proportion of sequences that contain the motif of interest. However, longer sequences, by chance, are more likely to contain motifs of interest. To adjust for this bias, we introduced length-matched control sequences. These random control sequences were extracted from a collection of the regions $5 \mathrm{~kb}$ upstream of annotated transcription starts of all RefSeq genes with annotated 5 UTRs. This set of promoter sequences was downloaded from the UCSC genome browser [51] website http://hgdownload.cse.ucsc.edu/downloads.html. Any sequence that overlapped with test sequences was excluded. Motif scan of the test and the control sequences was then performed using MatInspector [52] in the Genomatix suite (Genomatix $\mathrm{GmBH}$, Munich, Germany) with default settings. The numbers of test and control sequences either harbouring or lacking the expected motifs were recorded in a $2 \times 2$ contingency table. A Chi-square test was then performed to evaluate the significance of the motif enrichment. Motif enrichments of all 153 families of vertebrate motif matrices found in the Genomatix MatBase 7.0 database were calculated then ranked by the Chi-square test statistics.

\section{Additional material}

Additional file 1 More detailed information on methods and addi-

tional Tables and Figures.

\section{Authors' contributions}

ZSQ, JY, JS developed the methods and coded the software package. MH participated in the analysis of real datasets. CAM, SK, JY and AMC participated in the design and coordination of the study and reviewed the manuscript. JY and AMC also provided key biological insight that benefits the study design. All authors have read and approved the final manuscript.

\section{Acknowledgements}

We thank Dr. Terrence Barrette, Xuhong Cao and members of the Arul Chinnaiyan Laboratory for valuable suggestions and comments on earlier versions of the program and manuscript. We thank Dr. Tanya Teslovich and Ms. Jill Granger for critical reading of the manuscript. We thank Dr. Ghia Euskirchen of the Michael Synder Laboratory for providing us the list of identified peaks from the STAT1 ChIP-chip experiment. We are grateful to Dr. Olivier Elemento at Weill Cornell Medical College for his help with describing and using the ChIPseeger software. We are grateful to Dr. Hongkai Ji at Johns Hopkins University for his help with using the CisGenome software. CAM was supported by an NIH Ruth L. Kirschstein post-doctoral training grant and currently derives support from the American Association of Cancer Research Amgen Fellowship in Clinical/ Translational Research and the Canary Foundation and American Cancer Society Early Detection Postdoctoral Fellowship. JY was supported in part by Department of Defense New Investigator Award PC080665 and National Institutes of Health K99/R00 grant K99CA129565. AMC is supported by the Department of Defense Era of Hope grant BC075023 and the National Functional Genomics Center W81XWH-09-2-0014; National Cancer Institute SPORE in Prostate Cancer P50 CA69568, the Burroughs Wellcome Fund, and the Prostate Cancer Foundation. AMC is also an American Cancer Society Research Professor. JC, MH and ZSQ were supported in part by NIH grant R01HG005119. ZSQ was also support in part by NIH Comprehensive Cancer Center grant CA 46592 and Prostate SPORE grant CA69568.

\section{Author Details}

${ }^{1}$ Center for Statistical Genetics, Department of Biostatistics, School of Public Health, University of Michigan, 1415 Washington Heights, Ann Arbor, MI 481092029, USA, ${ }^{2}$ Center for Computational Medicine and Bioinformatics, University of Michigan Medical School, 100 Washtenaw Avenue, Ann Arbor, Ml 481092218, USA, ${ }^{3}$ Michigan Center for Translational Pathology, University of Michigan Medical School, 1400 E. Medical Center Drive, Ann Arbor, Ml 481090940, USA, ${ }^{4}$ Department of Pathology, University of Michigan Medical School, 1301 Catherine, Ann Arbor, Michigan 48109-0602, USA, 5Division of Hematology/Oncology, Robert H. Lurie Comprehensive Cancer Center, Northwestern University, 676 North St. Clair, Suite 1200, Chicago, IL 60611, USA , 6 Comprehensive Cancer Center, University of Michigan Medical School, 1500 E. Medical Center Drive, Ann Arbor, MI 48109-0944, USA, ${ }^{7}$ Department of Urology, University of Michigan Medical School, 1500 E. Medical Center Drive, Ann Arbor, MI 48109-0330, USA and ${ }^{8 H}$ Howard Hughes Medical Institute, 4000 Jones Bridge Road, Chevy Chase, MD 20815-6789, USA

Received: 20 July 2009 Accepted: 2 July 2010

Published: 2 July 2010

\section{References}

1. Orlando V, Paro R: Mapping Polycomb-repressed domains in the bithorax complex using in vivo formaldehyde cross-linked chromatin. Cell 1993, 75:1187-1198.

2. Solomon MJ, Larsen PL, Varshavsky A: Mapping protein-DNA interactions in vivo with formaldehyde: evidence that histone $\mathrm{H} 4$ is retained on a highly transcribed gene. Cell 1988, 53:937-947.

3. Massie CE, Mills IG: ChIPping away at gene regulation. EMBO Rep 2008, 9:337-343

4. Ren B, Robert F, Wyrick JJ, Aparicio O, Jennings EG, Simon I, Zeitlinger J, Schreiber J, Hannett N, Kanin E, et al:: Genome-wide location and function of DNA binding proteins. Science 2000, 290:2306-2309.

5. Lieb JD, Liu X, Botstein D, Brown PO: Promoter-specific binding of Rap1 revealed by genome-wide maps of protein-DNA association. Nat Genet 2001, 28:327-334.

6. Kim TH, Barrera LO, Ren B: ChIP-chip for genome-wide analysis of protein binding in mammalian cells. Curr Protoc Mol Biol 2007, Chapter 21: Unit 2113

7. Mardis ER: ChIP-seq: welcome to the new frontier. Nat Methods 2007 , 4:613-614. 
8. Barski A, Zhao K: Genomic location analysis by ChIP-Seq. J Cell Biochem 2009, 107:11-18

9. Schmidt D, Wilson MD, Spyrou C, Brown GD, Hadfield J, Odom DT: ChIPseq: Using high-throughput sequencing to discover protein-DNA interactions. Methods 2009

10. Johnson DS, Mortazavi A, Myers RM, Wold B: Genome-wide mapping of in vivo protein-DNA interactions. Science 2007, 316:1497-1502.

11. Robertson G, Hirst M, Bainbridge M, Bilenky M, Zhao Y, Zeng T, Euskirchen G, Bernier B, Varhol R, Delaney A, et al:: Genome-wide profiles of STAT1 DNA association using chromatin immunoprecipitation and massively parallel sequencing. Nat Methods 2007, 4:651-657.

12. Mikkelsen TS, Ku M, Jaffe DB, Issac B, Lieberman E, Giannoukos G, Alvarez P, Brockman W, Kim TK, Koche RP, et al:: Genome-wide maps of chromatin state in pluripotent and lineage-committed cells. Nature 2007, 448:553-560.

13. Barski A, Cuddapah S, Cui K, Roh TY, Schones DE, Wang Z, Wei G, Chepelev I, Zhao K: High-resolution profiling of histone methylations in the human genome. Cell 2007, 129:823-837.

14. Schones DE, Cui K, Cuddapah S, Roh TY, Barski A, Wang Z, Wei G, Zhao K: Dynamic regulation of nucleosome positioning in the human genome. Cell 2008, 132:887-898.

15. Chen $X, X u$ H, Yuan $P$, Fang F, Huss $M$, Vega VB, Wong E, Orlov YL, Zhang $W$, Jiang J, et al:: Integration of external signaling pathways with the core transcriptional network in embryonic stem cells. Cell 2008, 133:1106-1117.

16. Lefrancois P, Euskirchen GM, Auerbach RK, Rozowsky J, Gibson T, Yellman CM, Gerstein M, Snyder M: Efficient yeast ChIP-Seq using multiplex short-read DNA sequencing. BMC Genomics 2009, 10:37.

17. Welboren WJ, van Driel MA, Janssen-Megens EM, van Heeringen SJ, Sweep FC, Span PN, Stunnenberg HG: ChIP-Seq of ERalpha and RNA polymerase II defines genes differentially responding to ligands. Embo J 2009, 28:1418-1428.

18. Visel A, Blow MJ, Li Z, Zhang T, Akiyama JA, Holt A, Plajzer-Frick I, Shoukry $M$, Wright C, Chen F, et al:: ChIP-seq accurately predicts tissue-specific activity of enhancers. Nature 2009, 457:854-858.

19. Brunner AL, Johnson DS, Kim SW, Valouev A, Reddy TE, Neff NF, Anton E, Medina C, Nguyen L, Chiao E, et al:: Distinct DNA methylation patterns characterize differentiated human embryonic stem cells and developing human fetal liver. Genome Res 2009, 19:1044-1056.

20. Zhang Y, Liu T, Meyer CA, Eeckhoute J, Johnson DS, Bernstein BE, Nussbaum C, Myers RM, Brown M, Li W, Liu XS: Model-based Analysis of ChIP-Seq (MACS). Genome Biol 2008, 9:R137.

21. Valouev A, Johnson DS, Sundquist A, Medina C, Anton E, Batzoglou S, Myers RM, Sidow A: Genome-wide analysis of transcription factor binding sites based on ChIP-Seq data. Nat Methods 2008, 5:829-834

22. Ji H, Jiang H, Ma W, Johnson DS, Myers RM, Wong WH: An integrated software system for analyzing ChIP-chip and ChIP-seq data. Nat Biotechnol 2008, 26:1293-1300.

23. Jothi R, Cuddapah S, Barski A, Cui K, Zhao K: Genome-wide identification of in vivo protein-DNA binding sites from ChIP-Seq data. Nucleic Acids Res 2008, 360:5221-5231.

24. $\mathrm{Xu} \mathrm{H}$, Wei CL, Lin F, Sung WK: An HMM approach to genome-wide identification of differential histone modification sites from ChIP-seq data. Bioinformatics 2008, 24:2344-2349.

25. Kharchenko PV, Tolstorukov MY, Park PJ: Design and analysis of ChIP-seq experiments for DNA-binding proteins. Nat Biotechnol 2008 , 26:1351-1359

26. Rozowsky J, Euskirchen G, Auerbach RK, Zhang ZD, Gibson T, Bjornson R, Carriero N, Snyder M, Gerstein MB: PeakSeq enables systematic scoring of ChIP-seq experiments relative to controls. Nat Biotechnol 2009, 27:66-75.

27. Nix DA, Courdy SJ, Boucher KM: Empirical methods for controlling false positives and estimating confidence in ChIP-Seq peaks. BMC Bioinformatics 2008, 9:523

28. Spyrou C, Stark R, Lynch AG, Tavare S: BayesPeak: Bayesian analysis of ChIP-seq data. BMC Bioinformatics 2009, 10:299.

29. Choi H, Nesvizhskii Al, Ghosh D, Qin ZS: Hierarchical hidden Markov model with application to joint analysis of ChIP-chip and ChIP-seq data. Bioinformatics 2009, 25:1715-1721.

30. Fejes AP, Robertson G, Bilenky M, Varhol R, Bainbridge M, Jones $\mathrm{SJ}$ : FindPeaks 3.1: a tool for identifying areas of enrichment from massively parallel short-read sequencing technology. Bioinformatics 2008, 24:1729-1730

31. Albert I, Wachi S, Jiang C, Pugh BF: GeneTrack--a genomic data processing and visualization framework. Bioinformatics 2008, 24:1305-1306.

32. Laajala TD, Raghav S, Tuomela S, Lahesmaa R, Aittokallio T, Elo LL: A practical comparison of methods for detecting transcription factor binding sites in ChIP-seq experiments. BMC Genomics 2009, 10:618.

33. Li W, Meyer CA, Liu XS: A hidden Markov model for analyzing ChIP-chip experiments on genome tiling arrays and its application to p53 binding sequences. Bioinformatics 2005, 21(Suppl 1):i274-282.

34. Ji H, Wong WH: TileMap: create chromosomal map of tiling array hybridizations. Bioinformatics 2005, 21:3629-3636.

35. Munch K, Gardner PP, Arctander P, Krogh A: A hidden Markov model approach for determining expression from genomic tiling micro arrays. BMC Bioinformatics 2006, 7:239.

36. Huber W, Toedling J, Steinmetz LM: Transcript mapping with highdensity oligonucleotide tiling arrays. Bioinformatics 2006, 22:1963-1970.

37. Humburg P, Bulger D, Stone G: Parameter estimation for robust HMM analysis of ChIP-chip data. BMC Bioinformatics 2008, 9:343.

38. Euskirchen GM, Rozowsky JS, Wei CL, Lee WH, Zhang ZD, Hartman S, Emanuelsson O, Stolc V, Weissman S, Gerstein MB, et al: Mapping of transcription factor binding regions in mammalian cells by ChIP: comparison of array- and sequencing-based technologies. Genome Res 2007, 17:898-909.

39. Benjamini $Y$, Hochberg $Y$ : Controlling the false discovery rate: a pratical and powerful approach to multiple testing. J Royal Stat Soc B 1995, 57:289-300.

40. Shim H, Keles S: Integrating quantitative information from ChIP-chip experiments into motif finding. Biostatistics 2008, 9:51-65.

41. Consul PC: Generalized Poisson Distributions New York: Marcel Dekker 1989.

42. Johnson NL, Kotz S, Kemp AW: Univariate discrete distributions 2nd edition. New York: John Wiley \& Sons; 1992.

43. Siepel A, Bejerano G, Pedersen JS, Hinrichs AS, Hou M, Rosenbloom K, Clawson H, Spieth J, Hillier LW, Richards S, et al:: Evolutionarily conserved elements in vertebrate, insect, worm, and yeast genomes. Genome Res 2005, 15:1034-1050

44. Hu M, Yu J, Taylor JM, Chinnaiyan AM, Qin ZS: On the detection and refinement of transcription factor binding sites using ChIP-Seq data. Nucleic Acids Res 2010, 38:2154-2167.

45. Park PJ: Epigenetics meets next-generation sequencing. Epigenetics 2008, 3:

46. Down TA, Rakyan VK, Turner DJ, Flicek P, Li H, Kulesha E, Graf S, Johnson N, Herrero J, Tomazou EM, et al:: A Bayesian deconvolution strategy for immunoprecipitation-based DNA methylome analysis. Nat Biotechnol 2008, 26:779-785.

47. Mortazavi A, Williams BA, McCue K, Schaeffer L, Wold B: Mapping and quantifying mammalian transcriptomes by RNA-Seq. Nat Methods 2008, 5:621-628.

48. Valouev A, Johnson DS, Sundquist A, Medina C, Anton E, Batzoglou S, Myers RM, Sidow A: Genome-wide analysis of transcription factor binding sites based on ChIP-Seq data. Nat Methods 2008.

49. Yu J, Yu J, Mani RS, Cao Q, Brenner CJ, Cao X, Wang X, Wu L, Li J, Hu M, et al:: An integrated network of androgen receptor, polycomb, and TMPRSS2-ERG gene fusions in prostate cancer progression. Cancer Cell 17:443-454.

50. Rabiner LR: A Tutorial On Hidden Markov-Models and Selected Applications in Speech Recognition. Proceedings of the leee 1989, 77:257-286

51. Kent WJ, Sugnet CW, Furey TS, Roskin KM, Pringle TH, Zahler AM, Haussler D: The human genome browser at UCSC. Genome Res 2002, 12:996-1006.

52. Cartharius K, Frech K, Grote K, Klocke B, Haltmeier M, Klingenhoff A, Frisch $M$, Bayerlein M, Werner T: MatInspector and beyond: promoter analysis based on transcription factor binding sites. Bioinformatics 2005 , 21:2933-2942.

doi: 10.1186/1471-2105-11-369

Cite this article as: Qin et al., HPeak: an HMM-based algorithm for defining read-enriched regions in ChIP-Seq data BMC Bioinformatics 2010, 11:369 